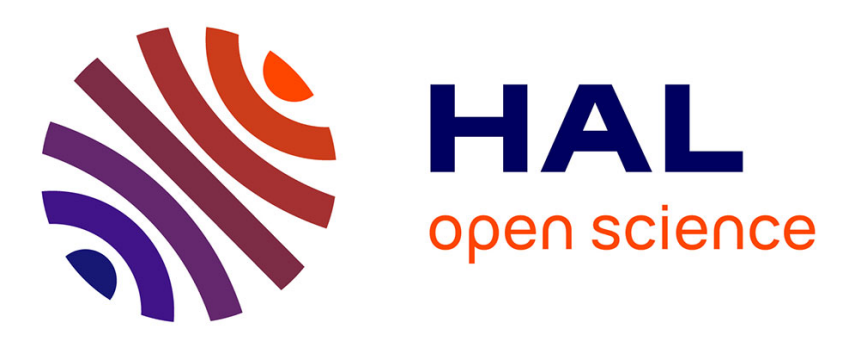

\title{
Posture recognition analysis during human-robot imitation learning
}

Hakim Guedjou, Sofiane Boucenna, Mohamed Chetouani

\section{To cite this version:}

Hakim Guedjou, Sofiane Boucenna, Mohamed Chetouani. Posture recognition analysis during humanrobot imitation learning. 2016 Joint IEEE International Conference on Development and Learning and Epigenetic Robotics (ICDL-EpiRob), Sep 2016, Cergy-Pontoise, France. 10.1109/DEVLRN.2016.7846817 . hal-01691767

\section{HAL Id: hal-01691767 https://hal.science/hal-01691767}

Submitted on 25 Jan 2018

HAL is a multi-disciplinary open access archive for the deposit and dissemination of scientific research documents, whether they are published or not. The documents may come from teaching and research institutions in France or abroad, or from public or private research centers.
L'archive ouverte pluridisciplinaire HAL, est destinée au dépôt et à la diffusion de documents scientifiques de niveau recherche, publiés ou non, émanant des établissements d'enseignement et de recherche français ou étrangers, des laboratoires publics ou privés. 


\section{Posture Recognition Analysis during Human-Robot Imitation Learning}

\author{
Hakim Guedjou \\ Institut des Systèmes Intelligents \\ et de Robotique \\ Sorbonne Univ., UPMC Univ. Paris 06, \\ UMR7222, F75005 Paris, France \\ Email : guedjou@isir.upmc.fr
}

\author{
Sofiane Boucenna \\ Équipes Traitement de l'Information \\ et Systèmes \\ CNRS UMR8051, ENSEA, UCP, \\ 95000 Cergy-Pontoise, France
}

\author{
Mohamed Chetouani \\ Institut des Systèmes Intelligents \\ et de Robotique \\ Sorbonne Univ., UPMC Univ. Paris 06, \\ UMR7222, F75005 Paris, France
}

\section{INTRODUCTION}

Imitation is a powerful paradigm for learning new actions and tasks and is currently investigated in several research domains such as developmental psychology or robotics [8]. For example, learning by demonstration is employed in robotics to teach new skills and tasks to robots [1] [2]. The dynamics of coupling of perception of one partner to the action of the other, and vice-and-versa, has been exploited to characterize social traits [9]. In particular, in the case of strong coupling such as imitation, research in developmental science has shown that imitation could be considered a social referencing mechanism [7]. Recently, identification of social traits during human-robot imitation learning has been successfully demonstrated. For example a social signature emerges from a neural network architecture and distinguishes typical children and children with Autism Spectrum Disorders (ASD). The key idea of this approach is to learn the mapping between the perception of postures performed by the children and the actions performed by the robot. A perception-action architecture based on neural network (PerAc [6]) is used to associate the perception with action [3]. Interestingly, this approach generalizes to person's identity recognition by the analysis of the dynamics of the social signature (novelty detection procedure) [5].

In this paper, we designed an analysis framework built upon the models and results on social signature detection. In particular, these previous works need further analysis. First, the impact of interaction time on imitation learning has not been explicitly investigated and here we report results on this issue. Secondly, the nature of posture may impact the learning and recognition rates. In this paper, we evaluate the posture recognition system performance by analyzing the recognition of the different postures.

\section{The IMITATION GAME AND THE LEARNING PROCESS}

In this study we use the same imitation architecture described in previous works [3], [6]. This imitation architecture considers a developmental approach. The robot is learning posture recognition during interactions with a human. During a learning phase, the robot performs random postures selected from five pre-designed postures (figure1)and the human is requested to imitate them. A neural network architecture is

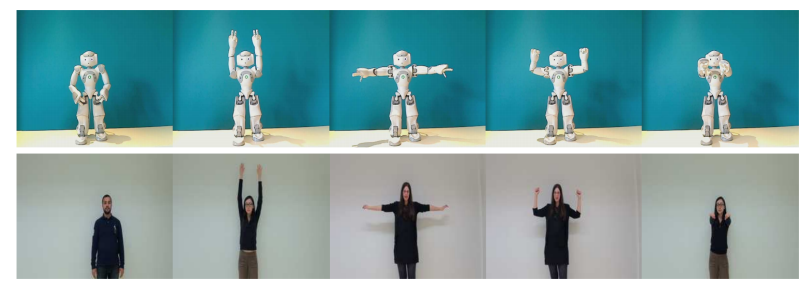

FIgURE 1. The robot and the human produce 5 basic postures : Neutral, Hands up, Flying, Muscles and Hands toward postures [3]

employed to learn the association between the perception of postures (visual input) and the robot's sensory state (actuators positions of the posture). During the test phase, the imitation procedure is reversed : the human performs random postures (from the pre-defined ones). The neural network architecture is employed to recognize the posture and the robot performs it. In this paper, we recorded several sessions of training and test phases that are analyzed. The postures are autonomously learned during a learning phase followed by a validation phase where the robot could make a posture recognition.

\section{EXPERIMENTAL PROTOCOL \& PRELIMINARY RESULTS}

The protocol was approved by the Pitié-Salpêtriére hospital ethics committee. All the parents received information on the experiment and gave written consent before the participation of their child. Fourteen children participated in the study. They were followed in the day-care setting for ASD of the PitieSalpêtriére hospital.

Each child performs the imitation game with a NAO robot (cf :secII). The game lasts for $3 \mathrm{~min}$ with less then $2 \mathrm{~min}$ for the learning phase. During this 2 minutes we record a set of images for an off-line processing and analysis. The images recorded are all annotated according to the postures the $\mathrm{NaO}$ is making during the learning phase. Therefore all the images are correctly labeled. Using the recorded data-set we perform two analyses. The first one focuses on individual strategy where we learn and test on the same one person, and the second one focuses on inter-individual strategy by following leaveone-subject-out methodology. For the individual strategy, we compute individual performance for different postures : For 
TABLE I

CONFUSION MATRIX OF THE FIVE POSTURES FOR THE INDIVIDUAL STRATEGY

\begin{tabular}{|c||c|c|c|c|c|}
\hline & Muscles & Flying & Neutral & Hands up & H toward \\
\hline \hline Muscles & 60.53 & 13.39 & 5.49 & 16.64 & 3.94 \\
\hline Flying & 8.27 & 65.90 & 14.40 & 5.58 & 5.85 \\
\hline Neutral & 2.12 & 5.32 & 77.75 & 8.87 & 5.92 \\
\hline Hands up & 23.23 & 3.50 & 7.44 & 60.93 & 4.91 \\
\hline Hands toward & 4.67 & 2.24 & 11.27 & 7.28 & 74.54 \\
\hline
\end{tabular}

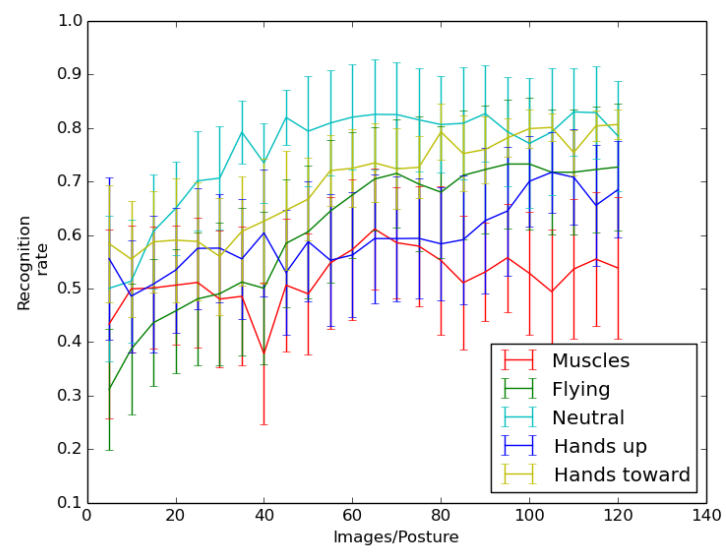

FIGURE 2. Recognition scores for the individual strategy

each child we use a part of the images recorded from him as learning images and the rest as testing ones. We change the number of learning images from 1 to 24 for each posture (which means from 5 to 120 repeated for each child to get a statistical significance). This analysis aims at understanding individual strategies and signatures, which is a required step for assessing children with Autism Spectrum Disorders. For the leave-one-subject-out methodology, we aim at analyzing generalization performance of imitation learning. Concretely, we learn models from all $\mathrm{N}-1$ children and test the model on the remaining one and we repeat this process $\mathrm{N}$ times. For each fold, we change the number of images from 1 to 24 for each posture (in total we go from 65 to 1560 images ).

The confusion matrix (table I) indicates the similarity in the postures for the individual strategy. Except for the "Muscles" and "Hands toward" postures, correct classification rates are more than $65 \%$. As expected, most of the confusions are obtained for similar postures such as "Hands up" and "Muscles". Figure 2 shows that for most of the postures, the number of images improve the performance expect of "Muscles" for which no significant improvement is observed.

Table II reports the generalization performance of the architecture with a leave-one-subject-out methodology. This experiments aims at evaluating inter-individual performances, i.e. using model learned from other individuals to recognize postures of a targeted individual. Results show that here again the number of images positively impacts the performance : from $43 \%$ to $94 \%$ recognition rates. In addition, this strategy
TABLE II

RECOGNITION SCORES DURING THE LEAVE-ONE-SUBJECT-OUT METHODOLOGY

\begin{tabular}{|c||c|c|c|}
\hline Nb of images/child & 5 & 10 & 120 \\
\hline \hline Muscles & $76 \%$ & $86 \%$ & $97 \%$ \\
\hline Flying & $32 \%$ & $49 \%$ & $91 \%$ \\
\hline Neutral & $40 \%$ & $55 \%$ & $94 \%$ \\
\hline Hands up & $28 \%$ & $43 \%$ & $91 \%$ \\
\hline Hands toward & $36 \%$ & $51 \%$ & $96 \%$ \\
\hline Average & $43 \%$ & $57 \%$ & $94 \%$ \\
\hline
\end{tabular}

allows to significantly improve the recognition of postures that are badly recognized in an individual approach (see "Muscles' performance) that is in conflict with results shown in [4]. One possible explanation is the existence of a higher complexity of facial expressions and the visual landmarks aren't enough informative for an exhaustive facial expression classification.

\section{CONCLUSION AND FUTURE WORKS}

This paper presents preliminary results on the analysis of posture recognition performance using imitation learning. In particular, we analyzed individual and inter-individual frameworks. The analysis clearly shows that the number of images improve the recognition scores for most of the postures. In addition, the inter-individual framework allows to reduce overfitting. The next step of this work is to evaluate the impact of posture recognition rates on identity recognition, which is based on a strong coupling between the human and the robot. We will also investigate differences between group of children (Typical/ASD) on posture and identity recognition and how the different postures influence this classification (Typical/ASD)

\section{REFERENCES}

[1] B. Argall, S. Chernova, M. Veloso, and B. Browning. A survey of robot learning from demonstration. Robotics and Autonomous Systems, $67: 469-483,2009$.

[2] A Billard, S. Callinon, R. Dillmann, and S. Schaal. Robot programming by demonstration. In B. Siciliano and O. (Eds.) Khatib, editors, Robot programming by demonstration, chapter 59. Springer, New York, 2008.

[3] S. Boucenna, S. Anzalone, E. Tilmont, D. Cohen and M. Chetouani. Learning of Social Signatures Through Imitation Game Between a Robot and a Human Partner. IEEE Transactions on Autonomous Mental Development, 6(3) :213-225, 2014/09//

[4] S. Boucenna, P. Gaussier, P Andry, and L. Hafemeister. A Robot Learns the Facial Expressions Recognition and Face/Non-face Discrimination Through an Imitation Game. International Journal os Social Robotics, $6: 633-652,2014 / 11 / /$

[5] S. Boucenna, Cohen D., P. Gaussier, A N Meltzoff, and M. Chetouani. Robots learn to recognize individuals from imitative encounters with people and avatars. Scientific Reports (Nature Publishing Group), srep19908, 2016.

[6] P. Gaussier, S. Zrehen PerAc : A neural architecture to control artificial animals. Robotics and Autonomous Systems, 16(2-4) :291-320, 1995//

[7] A. N. Meltzoff and M. Keith Moore. Early imitation within a functional framework: The importance of person identity, movement, and development. Infant Behavior and Development, 15(4) :479-505, 1992/12//

[8] A. N. Meltzoff, P. K. Kuhl, J. Movellan, T. J. Sejnowski. Foundations for a New Science of Learning. American Association for the Advancement of Science, 325(5938) :284-288, 2009//

[9] K. Yun, K. Watanabe, S. Shimojo. Interpersonal body and neural synchronization as a marker of implicit social interaction. Scientific Reports, srep00959, 2012// 